\title{
Correction to: A comparison of stage- specific all-cause mortality between testicular sex cordstromal tumors and germ cell tumors: results from the National Cancer Database
}

\author{
Kyle B. Zuniga ${ }^{1,2,3^{*}}$, Samuel L. Washington $1 \mathrm{I}^{1}$, Sima P. Porten ${ }^{1}$ and Maxwell V. Meng ${ }^{1}$
}

\section{Correction to: BMC Urol (2020) 20:40 \\ https://doi.org/10.1186/s12894-020-00609-2}

It was highlighted that the original article [1] contained the below errors in Tables 2, 3 and 4 and in the legends of Tables 3 and 4 . This Correction article shows the correct Tables and legends.

1) In Table 2, for Stage I - SCSTs the number for "uninsured" and "privately insured" was swapped. The distribution should be uninsured $=20(8 \%)$; privately insured $=169(65 \%)$

2) In Table 3, multivariable HR for Uninsured should read HR 2.31, 95\% CI 2.01-2.66. Additionally, Urban/Rural should read HR 1.13, 95\% CI 1.00-1.29. Among those with Stage II/III tumors, for percent of individuals in the patient's ZIP code without a high school diploma, the less than $7 \%$ group should read HR 0.67, 95\% CI 0.52-0.88. In Table 4, for stage II/ III, HR for income $>\$ 63 \mathrm{k}$ should read HR $0.79,95 \%$ CI 0.61-1.02. Additionally, for stage II/III, for percent of individuals in the patient's ZIP code without a high

The original article can be found online at https://doi.org/10.1186/s12894 020-00609-2

* Correspondence: kbz2102@columbia.edu

'Department of Urology, University of California, San Francisco, 550 16th Street, 6th Floor, San Francisco, CA 94158, USA

${ }^{2}$ Osher Center for Integrative Medicine, University of California, San Francisco, 1545 Divisadero Street, Suite 301, San Francisco, CA 94143, USA

Full list of author information is available at the end of the article school diploma, the less than $7 \%$ group should read HR 0.67, 95\% CI 0.52-0.88.

3) In Table 4, for stage II/III, HR for income $>\$ 63 \mathrm{k}$ should read HR 0.79, 95\% CI 0.61-1.02. Additionally, for stage II/III, for percent of individuals in the patient's ZIP code without a high school diploma, the less than $7 \%$ group should read HR 0.67, 95\% CI 0.52-0.88.

4) Table legend corrections: For Table 3, the definition of the abbreviation "IQR" is unnecessary. Table 4 was missing the following $-\mathrm{CI}=$ Confidence interval, GCTs $=$ Germ cell tumors, HR $=$ Hazard ratio, SCSTs $=$ Sex cord stromal tumors, ${ }^{*} p<0.05$, *** $p<0.01,{ }^{* * * *} p<0.001$

\section{Author details}

'Department of Urology, University of California, San Francisco, 550 16th Street, 6th Floor, San Francisco, CA 94158, USA. ${ }^{2}$ Osher Center for Integrative Medicine, University of California, San Francisco, 1545 Divisadero Street, Suite 301, San Francisco, CA 94143, USA. ${ }^{3}$ Vagelos College of Physicians and Surgeons, Columbia University Medical Center, 630 West 168th Street, New York, NY 10032, USA.

Published online: 17 July 2020

\section{Reference}

1. Zuniga KB, et al. A comparison of stage-specific all-cause mortality between testicular sex cordstromal tumors and germ cell tumors: results from the National Cancer Database. BMC Urol. 2020;20:40. https://doi.org/10.1186/ s12894-020-00609-2.

C The Author(s). 2020 Open Access This article is licensed under a Creative Commons Attribution 4.0 International License, which permits use, sharing, adaptation, distribution and reproduction in any medium or format, as long as you give appropriate credit to the original author(s) and the source, provide a link to the Creative Commons licence, and indicate if changes were made. The images or other third party material in this article are included in the article's Creative Commons licence, unless indicated otherwise in a credit line to the material. If material is not included in the article's Creative Commons licence and your intended use is not permitted by statutory regulation or exceeds the permitted use, you will need to obtain permission directly from the copyright holder. To view a copy of this licence, visit http://creativecommons.org/licenses/by/4.0/. The Creative Commons Public Domain Dedication waiver (http://creativecommons.org/publicdomain/zero/1.0/) applies to the data made available in this article, unless otherwise stated in a credit line to the data. 
Table 2 Stage-specific comparison of the sociodemographic and clinical characteristics of patients with SCSTs versus GCTs

\begin{tabular}{|c|c|c|c|c|c|c|}
\hline \multirow[t]{2}{*}{ Factor } & \multicolumn{2}{|l|}{ Stage I } & \multirow[b]{2}{*}{$\boldsymbol{p}$-value } & \multicolumn{2}{|l|}{ Stage $\|/\| \|$} & \multirow[b]{2}{*}{ p-value } \\
\hline & SCSTS & GCTs & & SCSTS & GCTs & \\
\hline N & 259 & 32,204 & & 21 & 9708 & \\
\hline Age at diagnosis, median (IQR) & $43(34,57)$ & $34(28,43)$ & $<0.001$ & $55(42,64)$ & $33(26,42)$ & $<0.001$ \\
\hline Diagnosis year & & & $<0.001$ & & & 0.38 \\
\hline 2004-2005 & $46(18 \%)$ & $6387(20 \%)$ & & $2(10 \%)$ & $1780(18 \%)$ & \\
\hline $2006-2007$ & $33(13 \%)$ & $6416(20 \%)$ & & $6(29 \%)$ & $1874(19 \%)$ & \\
\hline 2008-2009 & $45(17 \%)$ & $6528(20 \%)$ & & $5(24 \%)$ & $1942(20 \%)$ & \\
\hline 2010-2011 & 75 (29\%) & $6487(20 \%)$ & & $6(29 \%)$ & $1993(21 \%)$ & \\
\hline 2012-2013 & $60(23 \%)$ & $6386(20 \%)$ & & $2(10 \%)$ & $2119(22 \%)$ & \\
\hline Race/ethnicity & & & $<0.001$ & & & 0.62 \\
\hline Non-Hispanic White & $157(61 \%)$ & $25,125(78 \%)$ & & 17 (81\%) & $7266(75 \%)$ & \\
\hline Non-Hispanic Black & $47(18 \%)$ & $809(3 \%)$ & & $1(5 \%)$ & $311(3 \%)$ & \\
\hline Hispanic/Other & $52(20 \%)$ & $5672(18 \%)$ & & $3(14 \%)$ & $2000(21 \%)$ & \\
\hline Unknown & $3(1 \%)$ & $598(2 \%)$ & & $0(0 \%)$ & $131(1 \%)$ & \\
\hline Insurance & & & $<0.001$ & & & 0.087 \\
\hline Uninsured & $20(8 \%)$ & $3411(11 \%)$ & & $4(19 \%)$ & $1351(14 \%)$ & \\
\hline Private insurance & $169(65 \%)$ & $24,575(76 \%)$ & & $9(43 \%)$ & $6339(65 \%)$ & \\
\hline Medicaid/Medicare/other government insurance & $62(24 \%)$ & $3605(11 \%)$ & & $8(38 \%)$ & $1812(19 \%)$ & \\
\hline Unknown & $8(3 \%)$ & $613(2 \%)$ & & $0(0 \%)$ & $206(2 \%)$ & \\
\hline Income (per year) & & & 0.38 & & & 0.68 \\
\hline Less than $\$ 38 \mathrm{k}$ & $40(15 \%)$ & $3981(12 \%)$ & & $5(24 \%)$ & $1507(16 \%)$ & \\
\hline$\$ 38 k-62,999$ & $115(44 \%)$ & $15,408(48 \%)$ & & $10(48 \%)$ & $4791(49 \%)$ & \\
\hline$\$ 63 \mathrm{k}$ or greater & $100(39 \%)$ & $12,407(39 \%)$ & & $6(29 \%)$ & $3256(34 \%)$ & \\
\hline Unknown & $4(2 \%)$ & $408(1 \%)$ & & $0(0 \%)$ & $154(2 \%)$ & \\
\hline Percent in ZIP code without a high school degree & & & 0.69 & & & 0.75 \\
\hline $21 \%$ or greater & $37(14 \%)$ & $4450(14 \%)$ & & $5(24 \%)$ & $1711(18 \%)$ & \\
\hline $7-20.9 \%$ & $147(57 \%)$ & $17,658(55 \%)$ & & $12(57 \%)$ & $5355(55 \%)$ & \\
\hline Less than $7 \%$ & $71(27 \%)$ & $9716(30 \%)$ & & $4(19 \%)$ & $2496(26 \%)$ & \\
\hline Unknown & $4(2 \%)$ & $380(1 \%)$ & & $0(0 \%)$ & $146(2 \%)$ & \\
\hline Residence & & & 0.42 & & & 0.51 \\
\hline Metropolitan & $221(85 \%)$ & $26,877(84 \%)$ & & $16(76 \%)$ & $7934(82 \%)$ & \\
\hline Urban/rural & $38(15 \%)$ & $5327(17 \%)$ & & $5(24 \%)$ & $1774(18 \%)$ & \\
\hline Charlson-Deyo comorbidity score & & & 0.016 & & & $<0.001$ \\
\hline 0 & $237(92 \%)$ & $30,544(95 \%)$ & & $15(71 \%)$ & $9029(93 \%)$ & \\
\hline 1 or more & $22(9 \%)$ & $1660(5 \%)$ & & $6(29 \%)$ & $679(7 \%)$ & \\
\hline Stage & & & & & & 0.21 \\
\hline Stage I & 259 (100\%) & $32,204(100 \%)$ & & - & - & \\
\hline Stage II & - & - & & $9(43 \%)$ & $5469(56 \%)$ & \\
\hline Stage III & - & - & & $12(57 \%)$ & $4239(44 \%)$ & \\
\hline Treatment & & & $<0.001$ & & & $<0.001$ \\
\hline No orchiectomy & $0(0 \%)$ & $30(0.1 \%)$ & & $2(10 \%)$ & $634(7 \%)$ & \\
\hline Orchiectomy alone & $250(97 \%)$ & $16,519(51 \%)$ & & $10(48 \%)$ & $1106(11 \%)$ & \\
\hline Orchiectomy + adjuvant therapy & $9(4 \%)$ & $15,626(49 \%)$ & & $9(43 \%)$ & $7941(82 \%)$ & \\
\hline Other/unknown & $0(0 \%)$ & $29(0.1 \%)$ & & $0(0 \%)$ & $27(0.3 \%)$ & \\
\hline
\end{tabular}


Table 2 Stage-specific comparison of the sociodemographic and clinical characteristics of patients with SCSTs versus GCTs (Continued)

\begin{tabular}{|c|c|c|c|c|c|c|}
\hline \multirow[t]{2}{*}{ Factor } & \multicolumn{2}{|l|}{ Stage I } & \multirow[b]{2}{*}{$\boldsymbol{p}$-value } & \multicolumn{2}{|l|}{ Stage $\|/\| I \|$} & \multirow[b]{2}{*}{ p-value } \\
\hline & SCSTs & GCTs & & SCSTs & GCTs & \\
\hline \multirow[t]{2}{*}{ Last contact or death, months from diagnosis, median (IQR) } & $N=229$ & $N=28,855$ & $<0.001$ & $N=20$ & $N=8612$ & 0.002 \\
\hline & $41(22,62)$ & $53(29,80)$ & & $19(8,55)$ & $47(24,75)$ & \\
\hline \multirow[t]{2}{*}{ Time from diagnosis to death, median (IQR) } & $N=13$ & $N=747$ & & $N=14$ & $N=866$ & \\
\hline & $23(18,43)$ & $31(14,58)$ & 0.78 & $11(7,21)$ & $13(4,28)$ & 0.96 \\
\hline
\end{tabular}

GCTs $=$ Germ cell tumors, IQR $=$ Interquartile range, $\mathrm{SCST} s=$ Sex cord stromal tumors

Table 3 Univariable and multivariable Cox proportional hazards regression analysis on the association between sociodemographic and clinical characteristics and mortality of the overall cohort

\begin{tabular}{|c|c|c|}
\hline & Univariable HR (95\% Cl) & Multivariable $\mathrm{HR}(95 \% \mathrm{Cl})$ - Overall \\
\hline \multicolumn{3}{|l|}{ Tumor type } \\
\hline GCTs & Ref. & Ref. \\
\hline SCSTS & $2.96(2.03-4.33)^{* * *}$ & $1.68(1.13-2.49)^{*}$ \\
\hline Age (per 5-year increase) & $1.21(1.19-1.23)^{* * *}$ & $1.18(1.16-1.20)^{* * *}$ \\
\hline \multicolumn{3}{|l|}{ Race/ethnicity } \\
\hline Non-Hispanic White & Ref. & Ref. \\
\hline Non-Hispanic Black & $1.80(1.41-2.29)^{* * *}$ & $1.13(0.89-1.45)$ \\
\hline Hispanic/other & $1.27(1.13-1.43)^{* * *}$ & $1.14(1.01-1.30)^{*}$ \\
\hline \multicolumn{3}{|l|}{ Insurance } \\
\hline Private insurance & Ref. & Ref. \\
\hline Uninsured & $2.63(2.29-3.01)^{* * *}$ & $2.31(2.01-2.66)^{* * *}$ \\
\hline Medicaid/Medicare/other government insurance & $4.33(3.88-4.83)^{* * *}$ & $2.72(2.42-3.05)^{* * *}$ \\
\hline \multicolumn{3}{|l|}{ Income (per year) } \\
\hline$<\$ 38,000$ & Ref. & Ref. \\
\hline$\$ 38,000-\$ 62,999$ & $0.71(0.62-0.81)^{* * *}$ & $0.94(0.82-1.09)$ \\
\hline$>\$ 63,000$ & $0.44(0.38-0.50)^{* * *}$ & $0.76(0.63-0.92)^{* *}$ \\
\hline \multicolumn{3}{|l|}{ Percent in ZIP code without a high school diploma } \\
\hline$>21 \%$ & Ref. & Ref. \\
\hline $7-20.9 \%$ & $0.63(0.56-0.71)^{* * *}$ & $0.83(0.73-0.96)^{*}$ \\
\hline$<7 \%$ & $0.43(0.37-0.49)^{* * *}$ & $0.74(0.61-0.90)^{* *}$ \\
\hline \multicolumn{3}{|l|}{ Residence } \\
\hline Metropolitan & Ref. & Ref. \\
\hline Urban/rural & $1.49(1.33-1.67)^{* * *}$ & $1.13(1.00-\mathbf{1 . 2 9})$ \\
\hline \multicolumn{3}{|l|}{ Charlson-Deyo comorbidity score } \\
\hline 0 & Ref. & Ref. \\
\hline$\geq 1$ & $3.23(2.82-3.70)^{* * *}$ & $2.06(1.79-2.37)^{* * *}$ \\
\hline
\end{tabular}

$\mathrm{Cl}=$ Confidence interval, $\mathrm{GCTs}=$ Germ cell tumors, $\mathrm{HR}=$ Hazard ratio, SCSTs $=$ Sex cord stromal tumors

${ }^{*} p<0.05$

$* * p<0.01$

***p $<0.001$

${ }^{1}$ The following variables were included in the multivariable analysis: tumor type, age, diagnosis year, race/ethnicity, insurance, yearly income, percent in ZIP code without a high school diploma, residence, Charlson-Deyo comorbidity score 
Table 4 Multivariable Cox proportional hazards regression analysis on the association between sociodemographic and clinical characteristics and mortality by stage

\begin{tabular}{|c|c|c|}
\hline & Multivariable' HR $(95 \% \mathrm{Cl})$ - Stage I & Multivariable $\mathrm{HR}(95 \% \mathrm{Cl})$ - Stage $\|/ /\|$ \\
\hline \multicolumn{3}{|l|}{ Tumor type } \\
\hline GCTs & Ref. & Ref. \\
\hline SCSTS & $1.06(0.60-1.86)$ & $3.28(1.88-5.73)^{* * *}$ \\
\hline Age (per 5-year increase) & $1.23(1.20-1.26)^{* * *}$ & $1.13(1.10-1.16)^{* * *}$ \\
\hline \multicolumn{3}{|l|}{ Race/ethnicity } \\
\hline Non-Hispanic White & Ref. & Ref. \\
\hline Non-Hispanic Black & $1.18(0.80-1.72)$ & $1.13(0.81-1.56)$ \\
\hline Hispanic/other & $1.14(0.95-1.38)$ & $1.12(0.94-1.32)$ \\
\hline \multicolumn{3}{|l|}{ Insurance } \\
\hline Private insurance & Ref. & Ref. \\
\hline Uninsured & $2.58(2.08-3.21)^{* * *}$ & $2.07(1.72-2.50)^{* * *}$ \\
\hline Medicaid/Medicare/other government insurance & $3.15(2.64-3.75)^{* * *}$ & $2.31(1.97-2.70)^{* * *}$ \\
\hline \multicolumn{3}{|l|}{ Income (per year) } \\
\hline$<\$ 38,000$ & Ref. & Ref. \\
\hline$\$ 38,000-\$ 62,999$ & $0.92(0.74-1.15)$ & $0.96(0.79-1.16)$ \\
\hline$>\$ 63,000$ & $0.74(0.56-0.98)^{*}$ & $\mathbf{0 . 7 9}(0.61-1.02)$ \\
\hline \multicolumn{3}{|l|}{ Percent in ZIP code without a high school diploma } \\
\hline$>21 \%$ & Ref. & Ref. \\
\hline $7-20.9 \%$ & $0.87(0.70-1.07)$ & $0.80(0.67-0.97)^{*}$ \\
\hline$<7 \%$ & $0.80(0.61-1.06)$ & $\mathbf{0 . 6 7}(0.52-0.88)^{* *}$ \\
\hline \multicolumn{3}{|l|}{ Residence } \\
\hline Metropolitan & Ref. & Ref. \\
\hline Urban/rural & $1.18(0.98-1.42)$ & $1.09(0.91-1.29)$ \\
\hline \multicolumn{3}{|l|}{ Charlson-Deyo comorbidity score } \\
\hline 0 & Ref. & Ref. \\
\hline$\geq 1$ & $2.03(1.64-2.51)^{* * *}$ & $2.03(1.68-2.45)^{* * *}$ \\
\hline
\end{tabular}

$\mathrm{Cl}=$ Confidence interval, GCTs = Germ cell tumors, HR = Hazard ratio, SCSTs = Sex cord stromal tumors

${ }^{*} p<0.05$

$* * p<0.01$

***p $<0.001$

${ }^{1}$ The following variables were included in the multivariable analysis: tumor type, age, diagnosis year, race/ethnicity, insurance, yearly income, percent in ZIP code without a high school diploma, residence, Charlson-Deyo comorbidity score 